\title{
Peritoneal Serous Borderline Tumour with Ultra- Mutated NGS Profile Including POLE E, BRAF, RB1, HER2 and P53 Mutations : A Case Report.
}

Jean-Christophe Noel ( $\sim$ Jean-Christophe.Noel@erasme.ulb.ac.be )

ERASME UNIVERSITY HOSPITAL https://orcid.org/0000-0002-4395-6673

Antonio Buonomo

CHU Tivoli

Sarah Bouri

Hopital Erasme

Nicky D'Haene

Hopital Erasme

Xavier Catteau

CUREPATH

\section{Case Report}

Keywords: Peritoneal, serous borderline tumor, mutations, NGS, case report

Posted Date: June 8th, 2020

DOI: https://doi.org/10.21203/rs.3.rs-32311/v1

License: (a) (i) This work is licensed under a Creative Commons Attribution 4.0 International License. Read Full License 


\section{Abstract}

Background: Serous borderline tumors of the peritoneum are rare low-grade epithelial proliferation with a tubal-type differentiation occurring in the pelvis or abdomen without underlying tissue invasion that resemble to ovarian serous borderline tumors but by definition without ovrian or tubal involvement. To date, the mutation status in molecular biology of these tumours remains largely unknown.

Case presentation: Here we describe the case of a borderline peritoneal serous tumour which occurred in a 48 year old patient in whom an analysis of the mutation status by NGS technique identified a ultramutated profile including POLE E, BRAF, RB1, HER2 and p53 mutations.

Conclusions: To the best of our knowledge, this particular mutation status has never been described in this type of tumour. It could represent an early event with transient mutations, most of them will not fixate on the genome.

\section{Background :}

Serous borderline tumor of the peritoneum is a rare malignant low-grade epithelial proliferation with a tubal-type differentiation occurring in the pelvis or abdomen without underlying tissue invasion. By definition, in these tumors, both fallopian tubes and ovaries are grossly and microscopically normal or show only minimal tumoral cortical ovarian involvement less than $5 \times 5 \mathrm{~mm} 2(1,2,3,4,5)$. The histogenesis of this tumor is uncertain, but recent data hypothesize that it originates from preexisting mullerianosis and in particular proliferating endosalpingiosis (3). Contrary to ovarian borderline serous tumors where various genomic mutations (mainly BRAF or KRAS mutations) have been described, the genomic mutational status of peritoneal serous borderline tumor is unknown (6).

\section{Case Presentation :}

A 48 years-old patient, had been admitted in our department of gynaecology in April 2016 for recurrent abnormal uterine bleeding (intermenstrual or postmenopausal). She signed an informed consent according to the rule of the local ethic commitee. The ultrasonography depicted multiple uterine fibroids. The rest of gynaecological, clinical and biological examination including Ca-125, CEA and CA $19-9$ tests was within normal limit. Therefore, the pathological examination revealed firstly the presence of several benign leiomyomas and secondly of multiple small granular or nodular lesions less than $5 \mathrm{~mm}$ on serous surface of the uterus or tubo-ovarian ligament (Fig. 1). Microscopically, these serous nodules as well the small pelvic granulation which has also been resected, consisted on a proliferation of small papillae and epithelial clusters with psammoma bodies associated with a desmoplastic stroma (Figs. 2, 3). By immunohistochemistry the lesions were positive for PAX 8, WT1, EMA, BER-EP4, CA-125, ER and negative for CK 5-6, Calretinin, p53 and HER2. The proliferative index with Ki-67 antibody is low and estimated at about $5 \%$. According to these histological data, the diagnosis of peritoneal borderline serous tumor was 
made and consecutively a second look coelioscopy surgery was performed with left salpingooophorectomy and resection of any pelvic/omental lesions.

The microscopical examination of these lesions was identical with no evident invasion or ovarian involvement but in addition to the first examination various "mullerianosis" lesions and in particular endosalpingiosis were also observed (Fig. 4). A molecular profiling by NGS of the tumoral lesions was performed as we have previously described $(7,8)$. A ultra-mutated profile including both D440N mutation of the POLE E gene, S605N mutation of the gene BRAF, R358 mutation of the gene RB1, G776V of the gene HER2 and H178Y, S227F, G245S mutations of TP 53 gene. To date, the patient had normal clinical, biological and imagery follow-up with no recurrence.

\section{Discussion And Conclusions :}

Ultramutated tumors including POLE E mutations have been described in a variety of tumors including brain, stomach, pancreas and breast carcinoma. In the genital tract, they were also found in endometrioid carcinoma and carcinosarcoma of the endometrium and more rarely in endometrioid ovarian carcinoma (9). To the best of our knowledge, we described here the first case of borderline serous peritoneal tumor with POLE E mutation and ultramutated profile. Indeed, classically, in serous borderline tumors of the ovary only hotspot mutations including mainly KRAS, BRAF, NRAS, HER2 have been identified (6).

POLE E "proofreading" domain mutation is considered as an early event in sporadic tumors with secondary accumulations of others mutations including TP53 mutations which are in theory classically observed in high grade ovarian or peritoneal serous tumors. Some of these mutations are considered only as "passenger", unable to become fixed in the genome and to lead to biological changes. In addition, tumors with a high mutational tumoral burden including POLE E mutation are considered to have a better prognosis due in part to an effective anti-tumoral immune response (9). Interestingly, peritoneal serous borderline tumors have notably a favorable prognosis but recurrences can occur particularly if a progression to serous carcinoma is observed (4). The treatment of this tumor included hysterectomy and bilateral salpingo-oophorectomy with removal of any pelvic/omental tumor $(2,3,10)$. To date no recurrence were observed in our patient.

Peritoneal serous borderline tumors have lower response rates to conventional therapy. Although, as encountered here, the mutations BRAF gene, lead to constitutive BRAF kinase phosphorylation of MEK and ERK kinases and sustained MAPK pathway signaling. Therefore, targeted therapy with BRAF inhibitors can be an effective alternative treatment for these patients $(11,12,13)$.

In summary, we described the first case of perironeal serous borderline tumor with POLE E mutation and ultramutated profile. Classically, gynaecological tumors with this particular genetic profile have a more favourable prognosis but naturally, these preliminary data awaist confirmation.

\section{Abbreviations :}


BRAF : proto-oncogene B-RAF / v-Raf murine sarcoma viral oncogene homolog B ; Ca 19-9 : Carbohydrate antigen 19-9 ; CA-125 : cancer antigen 125 ; CEA : Carcinoembryonic Antigen ; CK 5-6 : Cytokeratins 5-6 ; EMA : Epithelial Membrane Antigen ; ERK : extracellular signal-regulated kinases ; HER 2 : Human Epidermal Growth Factor Receptor-2 ; KRAS : Kirsten Rat Sarcoma Virus ; MAP kinase : Mitogen-Activated Protein kinase; NGS : Next Generation Sequencing ; PAX8 : Paired box gene 8 ; POLE E : DNA polymerase epsilon catalytic subunit E; RB1 : Retinoblastoma, WT1 : Wilms tumor 1.

\section{Declarations}

\section{Author's contribution :}

$\mathrm{JCN}, \mathrm{XC}, \mathrm{SB}$ made the diagnosis and reviewed the literature. AB was the surgeon who treat the patient. ND made NGS. The authors read and approved the final manuscript.

\section{Funding :}

Not applicable

\section{Availability of data and materials :}

Not applicable.

\section{Consent to participate and for publication :}

The patient give and signed a " patient consent form » according to the local ethics committee and in accordance with Belgian and International law (Helsinki declaration).

\section{Competing interests :}

The authors declare that they have no competing interests.

\section{References}

1. Bell DA, Scully RE. Serous borderline tumours of the peritoneum. Am J Surg Pathol $1990 ; 14: 230-$ 239.

2. Biscotti CV, Hart CW. Peritoneal serous micropapillomatosis of low malignant potential (serous borderline tumours of the peritoneum). A clinicopathologic study of 17 cases. Am J Surg Pathol 1992 ; 16: 467-475. 
3. DeLair D, Soslow R. Key features of extrauterine pelvic serous tumours (fallopian tube, ovary and peritoneum). Histopathology $2012 ; 61: 329-330$.

4. Weir MM, Bell DA, Young RH. Grade 1 peritoneal serous carcinomas: a report of 14 cases and comparison with 7 peritoneal serous psammocarcinomas and 19 peritoneal serous borderline tumors. Am J Surg Pathol 1998 ; 22: 849-62.

5. Seidman JD, Savage J, Krishnan J, Vang R, Kurman RJ. Intratumoral Heterogeneity Accounts for Apparent Progression of Noninvasive Serous Tumors to Invasive Low-grade Serous Carcinoma: A Study of 30 Low-grade Serous Tumors of the Ovary in 18 Patients With Peritoneal Carcinomatosis. Int J Gynecol Pathol 2020; 39: 43-54.

6. Hunter SM, Anglesio MS, Ryland GL, Sharma R, Chiew YE, Rowley SM, Doyle MA, et al. Molecular profiling of low grade serous tumours identifies novel candidate driver genes. Oncotarget $2015 ; 6$ (35): 37663-37677.

7. Bienfait L, D'haene N, Catteau X, Noël JC. PIK3CA and p53 mutations by next generation sequencing in lymphoepithelioma-like carcinoma of the endometrium. Case Rep Pathol: $2018 ; 5894589$.

8. Catteau X, D'haene N, Noël J-C. Low grade malignant eccrine spiradenoma of the vulve : case report, review of the literature and discussion about the role of p53 and HPV. Diagn Pathol $2020 ; 15: 24$.

9. Rayner E, van Gool I, Palles C, Kearsey SE, Bosse T, Tomlinson I et al. A panoply of errors: polymerase proofreading domain mutations in cancer. Nat Rev Cancer 2016 ; 16: 71-81.

10. Hutton RL, Dalton SR. Primary peritoneal serous borderline tumors. Arch Pathol Lab Med 2007 ; 131 : 138-144.

11. Lavoué V, Rousselin A, Delplanque S, Pinsard M, Henno S, Foucher et al. Can new molecular profiles in epithelial ovarian cancer modify therapeutics. J Gynecol Obstet Hum Reprod 2017 ; 46: 107-112.

12. Mendivil AA, Tung PK, Bohart R, Bechtol K, Goldstein BH. Dramatic clinical response following dabrafenib and trametinib therapy in a heavily pretreated low grade serous ovarian carcinoma patient with BRAF V600E mutation. Gynecol Oncol Rep 2018 26: 41-44.

13. Gershenson DM. Low grade serous carcinoma of the ovary or peritoneum. Ann Oncol 2016 : i45-i49.

\section{Figures}




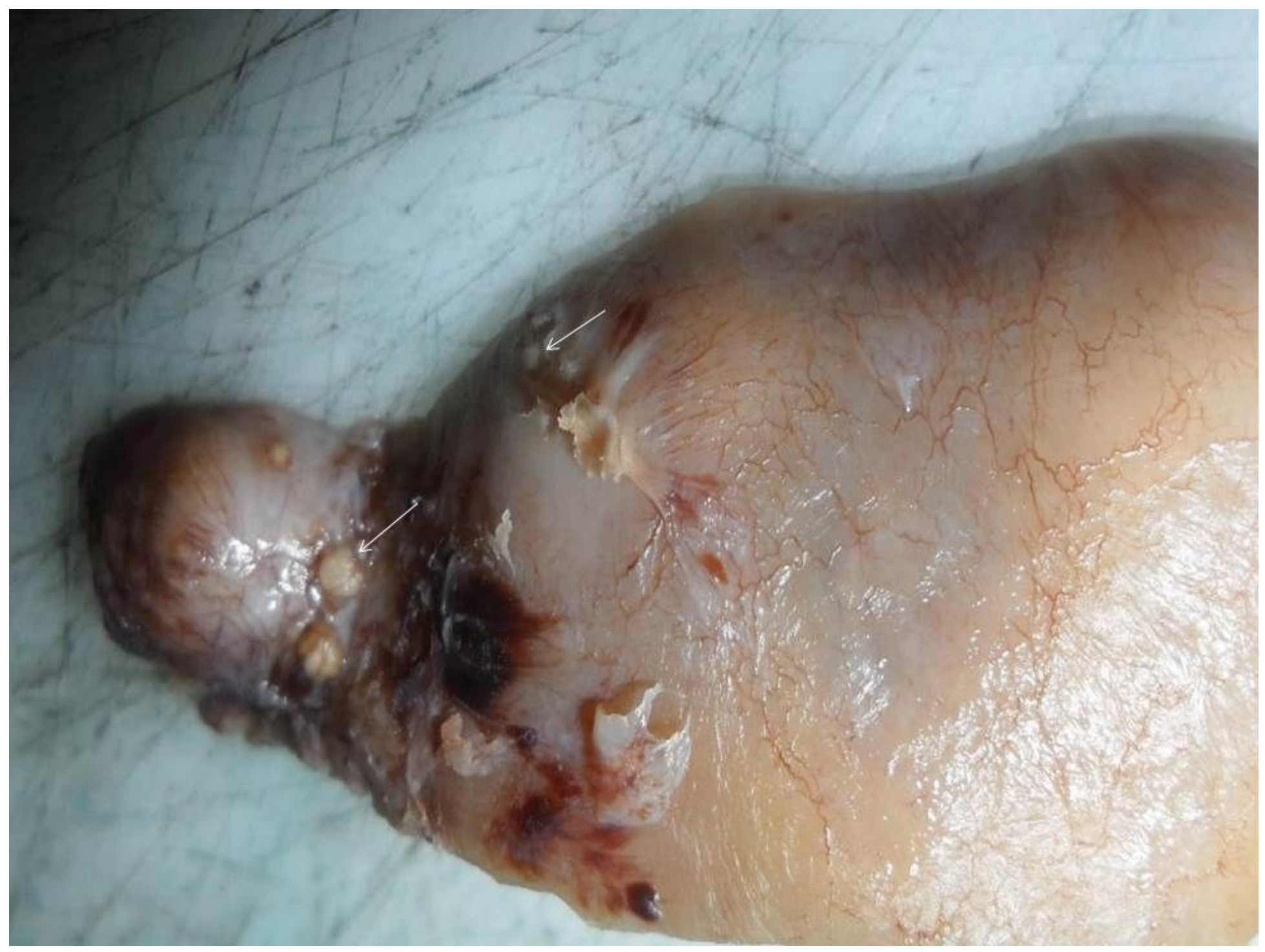

\section{Figure 1}

Macroscopic aspect of peritoneal serous borderline tumor. Multiple granular indurations or nodules ( $<1$ $\mathrm{cm})$ present here on serous uterine surface. 


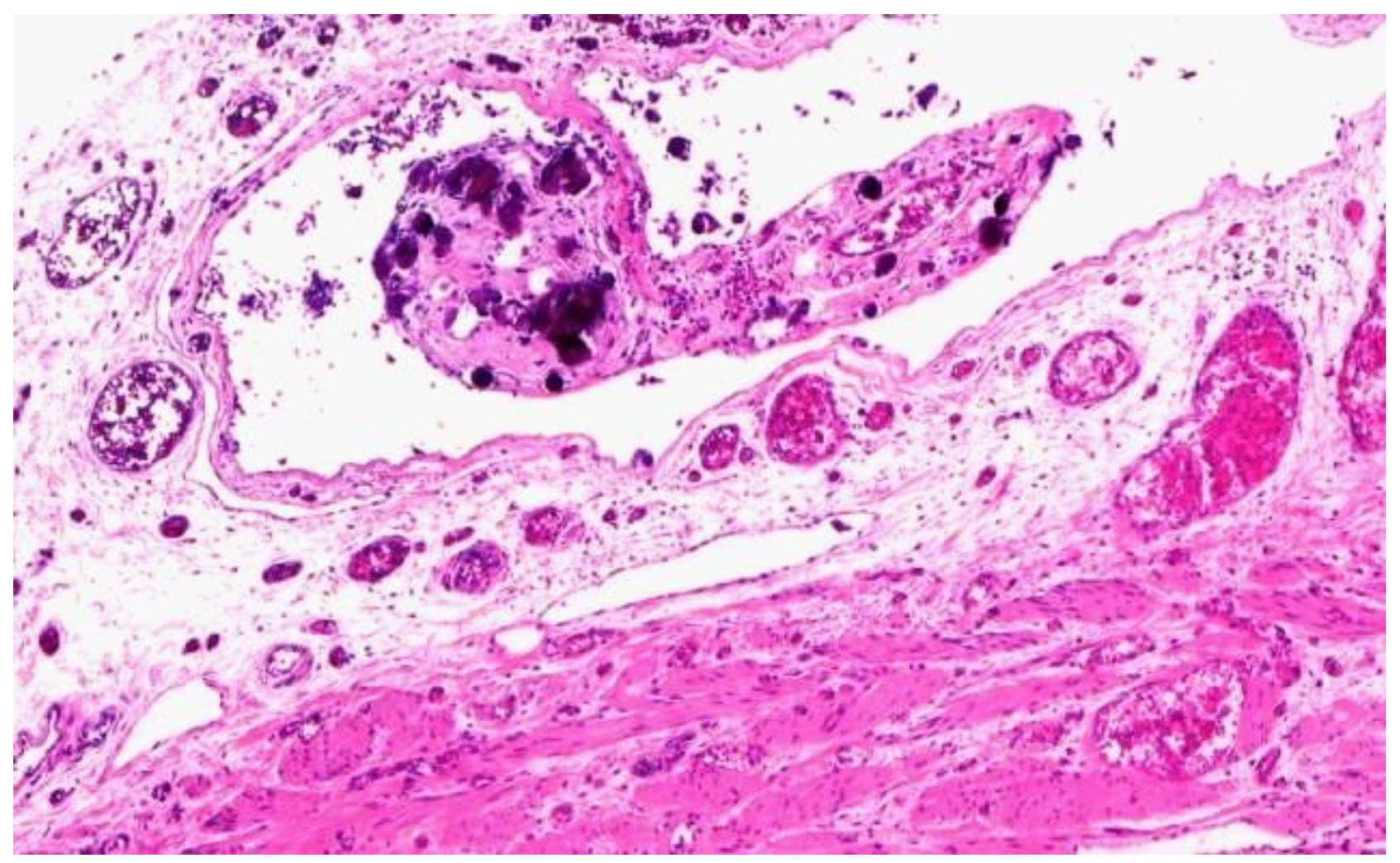

Figure 2

Microscopic aspects of peritoneal serous borderline tumor at low power view. Small papillae associated with numerous psammoma bodies embedded in a desmoplastic stroma ressembling to desmoplastic implants encountered in ovarian serous borderline tumor. Note the sharp demarcation from the underlying uterine smooth muscle here. 


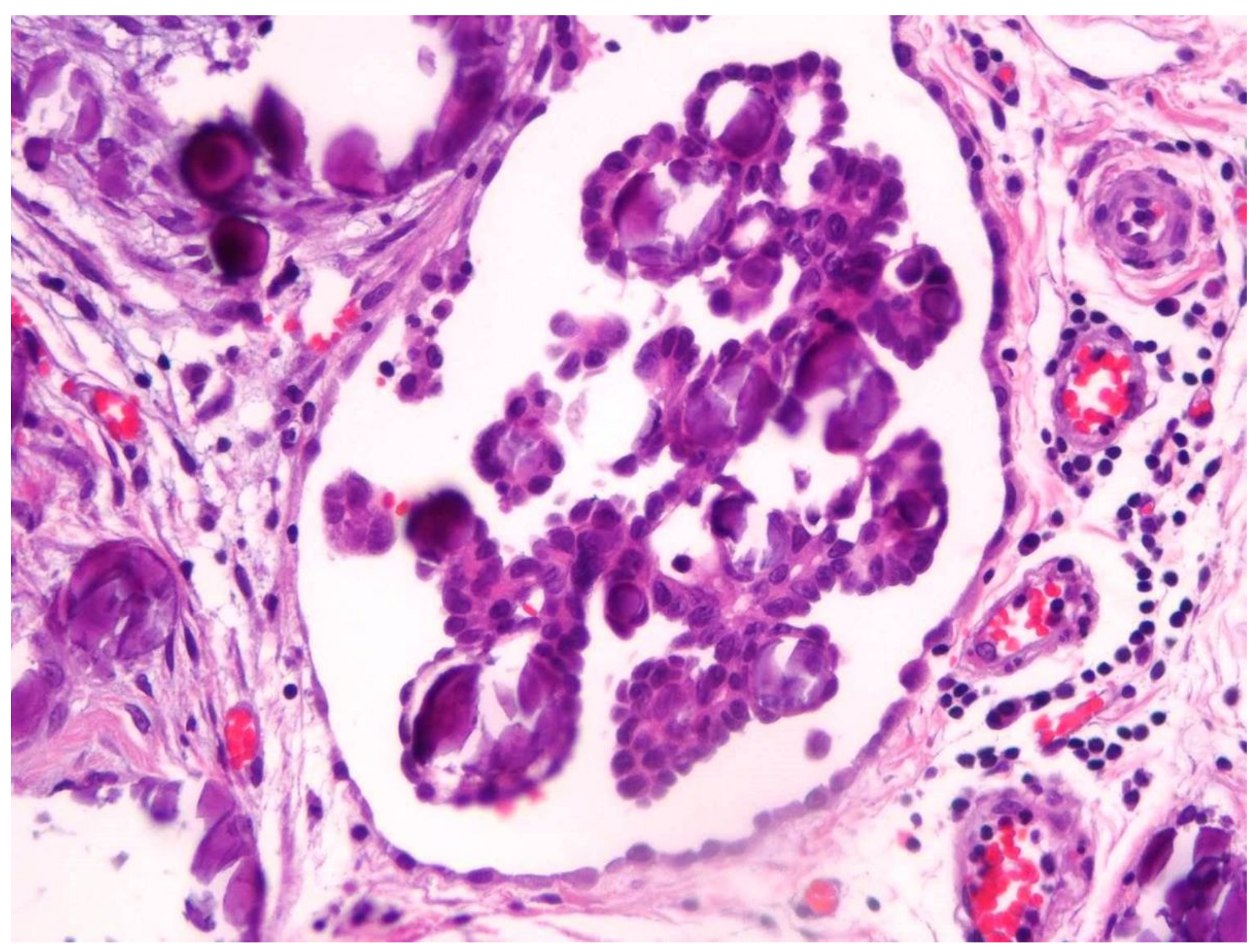

Figure 3

At high power view, papillae with tufting and budding are observed. The cuboidal cells are uniform and the mitotic activity is low. 


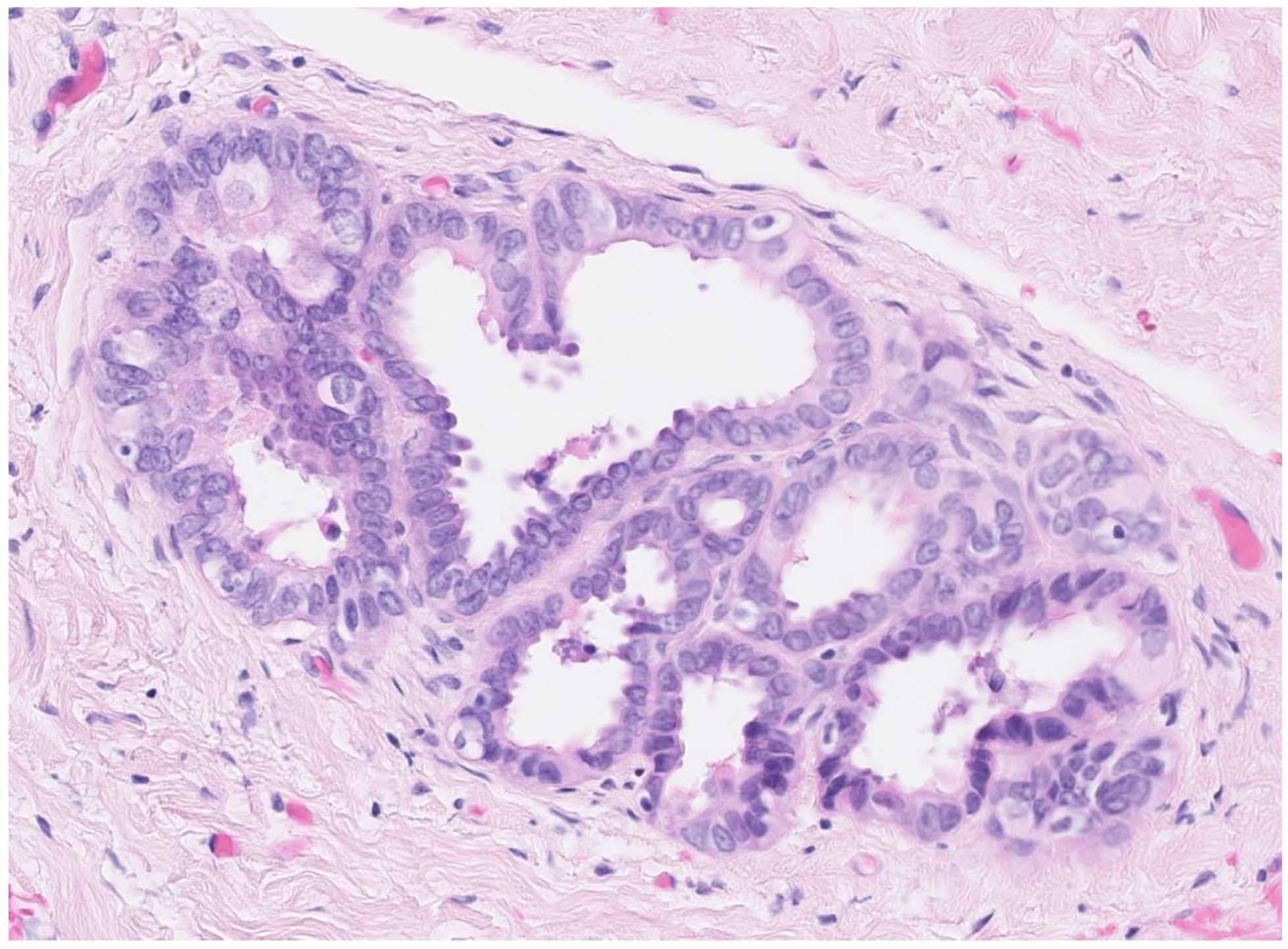

Figure 4

Associated endosalpingiosis is observed.

\section{Supplementary Files}

This is a list of supplementary files associated with this preprint. Click to download.

- CAREchecklistEnglish2013.pdf 